\title{
Exploring Elicitors of the Beneficial Rhizobacterium Bacillus amyloliquefaciens SQR9 to Induce Plant Systemic Resistance and Their Interactions With Plant Signaling Pathways
}

\author{
Gengwei Wu, ${ }^{1}$ Yunpeng Liu, ${ }^{2}$ Yu Xu, ${ }^{1}$ Guishan Zhang, ${ }^{2}$ Qirong Shen, ${ }^{1}$ and Ruifu Zhang ${ }^{1,2, \dagger}$ \\ ${ }^{1}$ Jiangsu Provincial Key Lab for Organic Solid Waste Utilization, National Engineering Research Center for Organic-based \\ Fertilizers, Jiangsu Collaborative Innovation Center for Solid Organic Waste Resource Utilization, Nanjing Agricultural \\ University, Nanjing, 210095, P.R. China; and ${ }^{2}$ Key Laboratory of Microbial Resources Collection and Preservation, Ministry of \\ Agriculture, Institute of Agricultural Resources and Regional Planning, Chinese Academy of Agricultural Sciences, Beijing \\ 100081, P.R. China
}

Accepted 3 January 2018.

\begin{abstract}
Beneficial rhizobacteria have been reported to produce various elicitors that induce plant systemic resistance, but there is little knowledge concerning the relative contribution of multiple elicitors from a single beneficial rhizobacterium on the induced systemic resistance in plants and the interactions of these elicitors with plant signaling pathways. In this study, nine mutants of the plant growth-promoting rhizobacterium Bacillus amyloliquefaciens SQR9 deficient in producing the extracellular compounds, including fengycin, bacillomycin $\mathrm{D}$, surfactin, bacillaene, macrolactin, difficidin, bacilysin, 2,3-butandiol, and exopolysaccharides, were tested for the induction of systemic resistance against Pseudomonas syringae pv. tomato DC3000 and Botrytis cinerea and the transcription of the salicylic acid, jasmonic acid, and ethylene signaling pathways in Arabidopsis. Deficiency in producing any of these compounds in SQR9 significantly weakened the induced plant resistance against these phytopathogens. These SQR9-produced elicitors induced different plant defense genes. For instance, the enhancement of 1,3-glucanase (PR2) by SQR9 was impaired by a deficiency of macrolactin but not surfactin. SQR9 mutants deficient in the lipopeptide and polyketide antibiotics remained only $20 \%$ functional for the induction of resistance-related gene transcription. Overall, these elicitors of SQR9 could act synergistically to induce plant systemic resistance against different phytopathogens through different signaling pathway genes, and the bacterial antibiotics are major contributors to the induction.
\end{abstract}

Gengwei Wu and Yunpeng Liu contributed equally to the article.

${ }^{\dagger}$ Corresponding author: Ruifu Zhang;

E-mail address: rfzhang@njau.edu.cn

Funding: This work was funded by the National Key Basic Research Program of China grant 2015CB150505, the National Key Research and Development Program grants 2016YFE0101100 and 2016YFD0200300, the 111 Project grant B12009, the National Natural Science Foundation of China grants 31572214, 31600088, and 31330069, the China Postdoctoral Science Foundation grant 2016M591297 and 2017T100118, and Key Projects of International Cooperation in Science and Technology Innovation grant 2016YFE0101100.

*The $\boldsymbol{e}$-Xtra logo stands for "electronic extra" and indicates that two supplementary figures and six supplementary tables are published online.

(c) 2018 The American Phytopathological Society
Agricultural production is encountering great challenges from plant pathogens, which have caused worldwide significant yield decreases. Application of plant growth-promoting rhizobacteria (PGPR) has been known to be an efficient way to suppress plant pathogens. One of the mechanisms of PGPR in exerting their biocontrol function is the induced systemic resistance (ISR) of plants against a broad spectrum of phytopathogens in aboveground plant tissues (Glazebrook 2005; Meziane et al. 2005; Ryu et al. 2003; Yi et al. 2013), which has been described as the "activation of the host plant's physical or chemical defenses by an inducing agent" (Kloepper 1993). Root-colonized PGPR induce systemic resistance by producing a range of secondary metabolites, which are called elicitors. After the elicitors are sensed, the jasmonic acid (JA), ethylene (ET), and salicylic acid (SA) signaling pathways are activated to trigger plant resistance. Characterization of bacterial elicitors is meaningful for understanding the priming of plant defenses against phytopathogens and, consequently, guiding agricultural production.

So far, many elicitors produced by PGPR have been identified and characterized. Pseudomonas elicitors, such as 2,4diacetylphloroglucinol and $\mathrm{N}$-acylated-l-homoserine lactones, have been well-characterized at the molecular level (Iavicoli et al. 2003; Schuhegger et al. 2006). Volatile organic compounds (VOCs) produced by Bacillus subtilis GB03 and $\mathrm{Ba}$ cillus amyloliquefaciens IN937a have been reported to trigger the activation of ET/JA-responsive gene PDF1.2 (Ryu et al. 2004; Sharifi and Ryu 2016). Similarly, another volatile compound, dimethyl disulfide, produced by Bacillus cereus $\mathrm{C} 1 \mathrm{~L}$ plays an important role in inducing resistance to plant fungal diseases in tobacco and corn plants (Huang et al. 2012). Fengycin and surfactin, produced by $B$. subtilis strains, exhibit a significant ISR-mediated protective effect on bean plants and could activate the lipoxygenase pathway in tomato (Ongena et al. 2007). PeBA1 protein produced by $B$. amyloliquefaciens NC6 could induce systemic resistance against a broad spectrum of pathogens, including Tobacco mosaic virus (TMV) and the fungal pathogen Botrytis cinerea, since SA-responsive PRla, $P R 1 b, P R 5$, and $P A L$ as well as JA-responsive $P D F 1.2$ and COI1 were up-regulated upon treatment with PeBA1 (Wang et al. 2016). These studies indicated that SA, JA, and ET signaling pathways are involved in corresponding elicitor processes. 
However, most of the previous studies have focused on a single or few elicitors from rhizobacteria (Cawoy et al. 2015; Pieterse et al. 2014). One bacterium is usually equipped with multiple potential elicitors to activate plant systemic resistance. For example, a plant-beneficial rhizobacterium, Bacillus amyloliquefaciens SQR9, produced a range of secondary metabolites, such as the surfactin, fengycin, bacillomycin, bacillaene, macrolactin, difficidin, bacilysin, indole-3-acetic acid (IAA), and 2,3-butanediol (Li et al. 2014; Shao et al. 2015). Several of these compounds have been reported to be elicitors of plant resistance, such as surfactin, fengycin, and 2,3-butanediol (Ongena et al. 2007; Ryu et al. 2004). A comprehensive evaluation of these potential elicitors from one plant-beneficial rhizobacterium to coordinate and contribute to the overall ISR response of the plant host is generally lacking. Moreover, SA, JA, and ET signaling pathways are involved in plant ISR responses, and the interactions of these multiple elicitors and plant signaling pathways are in need of systemic exploration from a holistic view. Therefore, the objective of this study is to i) systematically characterize and evaluate the relative contributions of multiple elicitors from a single rhizobacterium to the overall ISR and ii) explore their interactions with the plant signaling pathways.

To achieve these objectives, a well-studied plant-beneficial rhizobacterium, B. amyloliquefaciens SQR9, and the model plant Arabidopsis thaliana (L.) Columbia (Col-0) were used for this study. Strain SQR9 has been demonstrated to possess efficient plant growth-promoting and biocontrol activities ( $\mathrm{Li}$ et al. 2014; Liu et al. 2016; Shao et al. 2015; Xu et al. 2013); SQR9 exerted its plant-beneficial effects through sensing the root-secreted signals (Liu et al. 2014, 2017) and producing secondary metabolites to affect the plant host (Chen et al. 2016, 2017). In this study, we demonstrated that SQR9 produced secondary metabolites that acted as elicitors in inducing the systemic resistance of Arabidopsis against Pseudomonas syringae pv. tomato DC3000 and Botrytis cinerea, and the lipopeptides, polyketides, and dipeptide antibiotics contributed the major roles for the ISR. Elicitors have specific effects on the induction of plant defense pathways and against different phytopathogens.

\section{RESULTS}

\section{Plant-beneficial rhizobacterium}

B. amyloliquefaciens SQR9 induced plant systemic resistance.

Infection of the phytopathogens $P$. syringae pv. tomato DC3000 and Botrytis cinerea were used as indicators to test whether SQR9 induces resistance in Arabidopsis. The subsequent quantification of pathogens was based on plate counting and disease severity for DC3000 and Botrytis cinerea, respectively. The results showed that inoculation with SQR9 led to a significant decrease of DC3000 infection (190-fold at 4 days and 40-fold at 6 days postinoculation (dpi) with DC3000) (Fig. 1A; Supplementary Table S1). At 4 and 6 dpi with Botrytis cinerea, the disease incidence decreased by 33.3 and $23.1 \%$, respectively, and the area under disease progress curve (AUDPC) decreased by 25.8 and $28.4 \%$, respectively, in plants treated with SQR9 compared with the control (Fig. 1B and C; Supplementary Table S2).

SQR9 activated plant SA, JA, and ET signaling pathways.

To investigate whether the SA, JA, or ET signaling pathways are involved in the ISR response activated by SQR9-produced elicitors, the contents of SA, JA, and ET in plants were measured. The plants without inoculation with SQR9 served as control (CK) plants. We observed that root contents of SA, JA, and ET increased the most (1.4-fold, twofold, and 1.4-fold of those in CK, respectively) after inoculation with SQR9 for 1 day, after which the contents of SA and JA have quickly decreased but the increase of ET content lasted longer; ET content in SQR9-inoculated root was still significantly higher than that in the CK root after 4 days (Fig. 2). However, the shoots showed a slower response than the roots did. The content of SA and JA in shoots gradually increased until reaching 1.3fold higher than the $\mathrm{CK}$ at $4 \mathrm{dpi}$, while ET increased to 1.2-fold at 2 dpi (Fig. 2; Supplementary Table S3). These results indicate that the accumulation of hormones in local tissues is faster than in those distal.

Furthermore, the transcription levels of genes involved in the SA, JA, and ET signaling in leaves were evaluated. Generally, the tested genes involved in all three signaling pathways were activated by SQR9 (Fig. 3; Supplementary Table S4). For SA signaling, the NPR1 protein, which is a receptor of SA and a transcriptional coregulator, increased to the highest value (8.4fold) at $6 \mathrm{~h}$ postinoculation (hpi) (Fig. 3A). The transcription of SA-inducible marker defense protein PR1 increased and reached the highest value (3.7-fold) at nearly the same time as NPR1 (Fig. 3A). PR2 and PR5 responded to SQR9 inoculation faster but at a lower level than PR1. For JA signaling, the transcription of allene oxide synthase (AOS), the key JA biosynthesis enzyme, increased to its highest level (5.7-fold) $1 \mathrm{hpi}$ (Fig. 3B), while the transcription of COI1 and the downstream transcription factor MYC2 reached their highest levels (sevenfold and 4.7-fold) at $3 \mathrm{~h}$ and $12 \mathrm{hpi}$, respectively (Fig. 3B). ERF1, a downstream regulator of the ET signaling pathway, was up-regulated to its highest level of 3.8 -fold at 3 to $6 \mathrm{hpi}$; as a consequence, HEL (pathogenesis-related protein 4), a defense
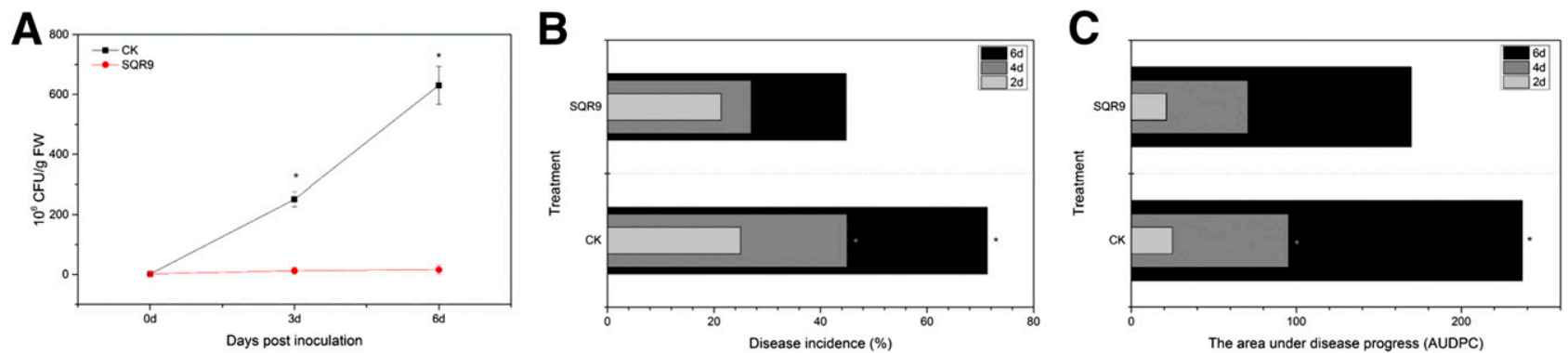

Fig. 1. Induction of systemic resistance by Bacillus amyloliquefaciens SQR9 in Arabidopsis. Five-week-old seedlings were inoculated with SQR9. Four days later, leaves were injected or sprayed with Pseudomonas syringae pv. tomato DC3000 or Botrytis cinerea, respectively. A, Percentage of DC3000 colonyforming units in the leaves of Arabidopsis plants inoculated by SQR9. B, Disease incidence of Arabidopsis caused by Botrytis cinerea. C, The area under the disease-progress curve (AUDPC) of Arabidopsis caused by Botrytis cinerea. Symptoms were scored at 2, 4, and 6 days postinoculation. Disease incidence and the AUDPC were calculated following the method described previously (Jeger and Viljanen-Rollinson 2001; Madden and Hughes 1999). The values are the means \pm the standard deviation of 12 replicates. An asterisk $(*)$ indicates statistically significant differences between plants inoculated with SQR9 and control plants without inoculation $(P \leq 0.05)$. 
gene under the regulation of the ET pathway, reached its highest level (fourfold) at the same time (Fig. 3C). Moreover, transcription of CHIB and PDF1.2 reached the highest levels (11.7-fold and 5.5-fold) at $12 \mathrm{hpi}$ (Fig. 3D). These results indicate that all three signaling pathways in Arabidopsis were activated by inoculation with SQR9.

\section{SQR9 produced multiple elicitors}

to induce plant systemic resistance.

To identify the SQR9-produced compounds that elicit a systemic resistance in Arabidopsis, SQR9 mutants deficient in the production of each potential elicitor (antibiotics, growthpromoting compounds, and exopolysaccharides) were tested for their function in inducing systemic resistance. The descriptions of these SQR9 mutants are shown in Table 1.

In brief, the results showed that mutations in the production of lipopeptide and polyketide antibiotics $(\Delta s f p$ in the $s f p$ gene, which is required for phosphopantetheine translocation and, thus, necessary for synthesis of all these antibiotics) caused a sharp decrease in the ability of SQR9 to induce plant resistance against $P$. syringae pv. tomato DC3000 and Botrytis cinerea (Fig. 4). For DC3000, the mutant $\Delta s f p$ showed only a quarter of the ability of the wild-type strain to trigger plant resistance against DC3000 (Fig. 4A). Even more, induction of plant resistance against Botrytis cinerea was completely blocked when $s f p$ was knocked out in SQR9 (Fig. 4B). For single antibiotics,
A

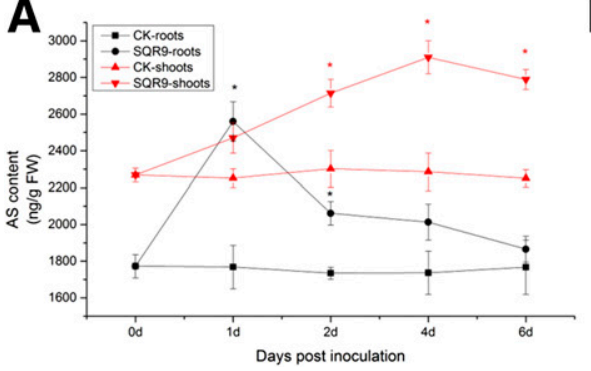

B

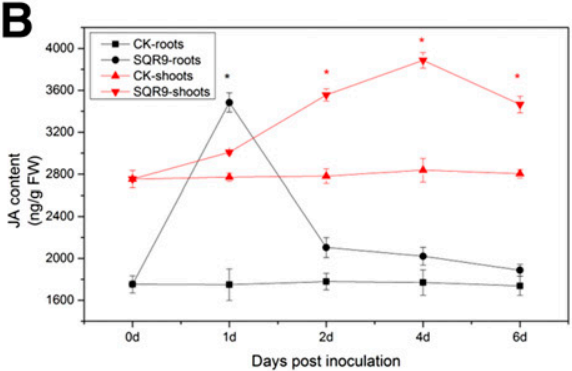

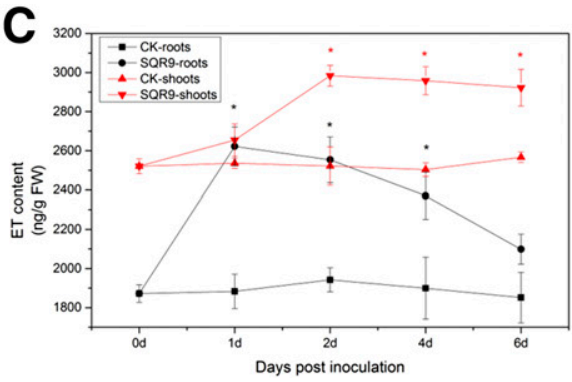

Fig. 2. Plant hormone accumulation in Arabidopsis treated with SQR9. Ten-day-old seedlings were treated with SQR9, and tissue sample of roots or shoots were harvested at the indicated timepoints. A, Salicylic acid content in Arabidopsis. B, Jasmonic acid content in Arabidopsis. C, Ethylene content in Arabidopsis. The values are the means \pm standard deviation of 12 replicates. An asterisk $(*)$ indicates a statistically significant difference $(P \leq 0.05)$.

A
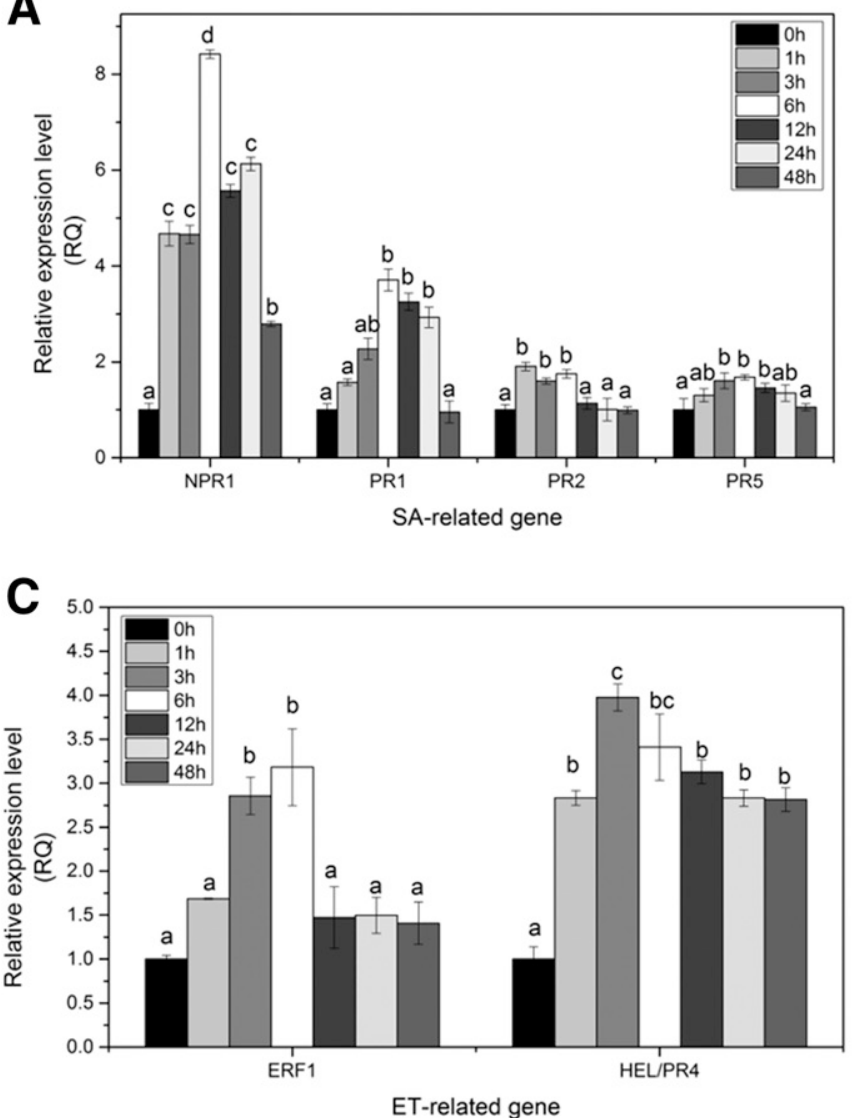

B

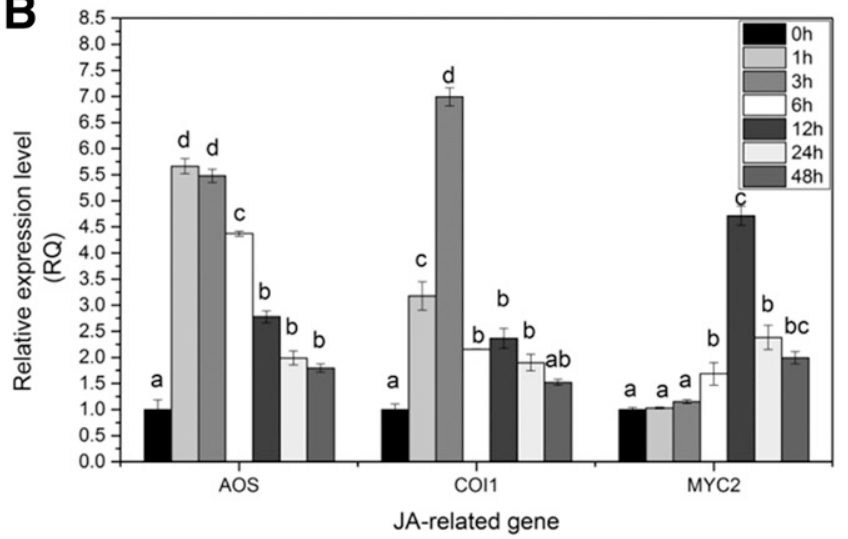

D

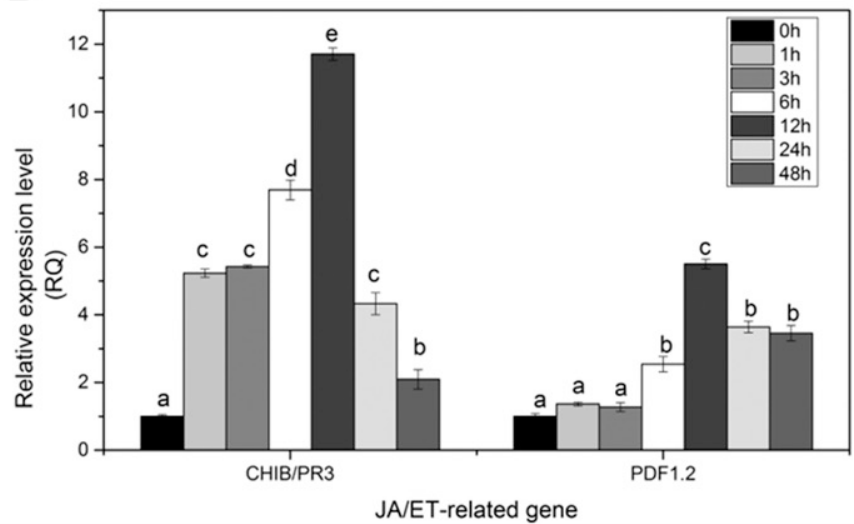

Fig. 3. Transcription of defense-related genes in Arabidopsis in response to SQR9 inoculation. Ten-day-old seedlings were treated with SQR9, and shoot samples were harvested at the indicated timepoints for extracting total RNA. A, The results of real-time quantitative polymerase chain reaction analysis of salicylic acid (SA)-related, B, jasmonic acid (JA)-related, C, ethylene (ET)-related, and D, JA/ET-related gene transcript levels in response to SQR9 at different times postinoculation. The values are the means \pm standard deviation of 12 replicates. Different letters above the bars indicate significant differences $(P \leq 0.05)$. 
mutants deficient in surfactin, bacillomycin $\mathrm{D}$, fengycin, or macrolactin production showed half the ability of SQR9 to induce plant resistance against DC3000, while other antibiotics, such as bacillaene, difficidin, and bacilysin, did not show remarkable contributions (Fig. 4A). For inducing resistance against Botrytis cinerea, mutations of each antibiotic production showed significant reductions compared with the wild-type strain at 4 days postinfection (Fig. 4B); among them, bacillomycin $\mathrm{D}$, surfactin, difficidin, bacillaene, and bacilysin showed significantly higher contributions to plant resistance against Botrytis cinerea than other antibiotics (Fig. 4B). These results indicate that all these antibiotics are elicitors of plant systemic resistance with specificity against different pathogens.
In addition to the antibiotics, SQR9-produced IAA contributed to plant ISR, since an IAA-deficient mutant $(\Delta y s n E)$ exhibits a significantly reduced ability to induce plant resistance against Botrytis cinerea but no significant effect on plant resistance against DC3000 was observed (Fig. 4B). The SQR9 mutants $\Delta a l s D$ and $\Delta e p s D$, deficient for 2,3-butanediol and exopolysaccharide production, respectively, showed significantly reduced abilities to induce plant resistance against both DC3000 and Botrytis cinerea (Fig. 4B).

\section{Correspondence analysis of SQR9 elicitors and plant defense signaling pathways.}

To evaluate the contribution of SQR9-produced elicitors to these plant signaling pathways, the transcription of these

Table 1. Bacterial and fungal strains used in this study ${ }^{\mathrm{a}}$

\begin{tabular}{|c|c|c|}
\hline Strain & Description & Source \\
\hline Bacillus amyloliquefaciens SQR9 & A plant growth-promoting rhizobacteria strain, isolated from cucumber rhizosphere & Cao et al. 2011 \\
\hline B. amyloliquefaciens SQR9Dbam::Tc ${ }^{r}$ & Deficient in producing bacillomycin $\mathrm{D}$ & Xu et al. 2013 \\
\hline B. amyloliquefaciens SQR9 4 fen::Tc ${ }^{r}$ & Deficient in producing fengycin & Xu et al. 2013 \\
\hline 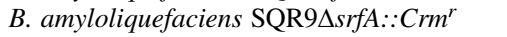 & Deficient in producing surfactin & Li et al. 2014 \\
\hline B. amyloliquefaciens SQR9 $\mathrm{Sbae}:: \mathrm{Crm}^{r}$ & Deficient in producing bacillaene & This study \\
\hline B. amyloliquefaciens SQR9 $\mathrm{S} d \mathrm{fn}:: \mathrm{Crm}^{r}$ & Deficient in producing difficidin & This study \\
\hline B. amyloliquefaciens SQR9 $\Delta m \ln 2:: \mathrm{Crm}^{r}$ & Deficient in producing macrolactin & This study \\
\hline 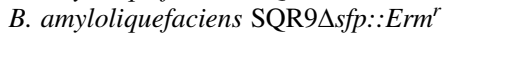 & $\begin{array}{l}\text { Deficient in producing bacillomycin D, fengycin, surfactin, bacillaene, difficidin, } \\
\text { macrolactin, and bacilysin }\end{array}$ & Li et al. 2014 \\
\hline B. amyloliquefaciens SQR9 $\mathrm{bac}:: \mathrm{Crm}^{r}$ & Deficient in producing bacilysin & This study \\
\hline 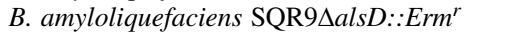 & Deficient in producing 2,3-butanediol & This study \\
\hline B. amyloliquefaciens SQR94epsD::Crm ${ }^{r}$ & Deficient in producing extracellular polysaccharides & This study \\
\hline 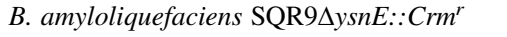 & Reduced indole-3-acetic acid synthesis & Shao et al. 2015 \\
\hline Pseudomonas syringae pv. tomato DC 3000 & A bacterial pathogen strain & \\
\hline Botrytis cinerea & A fungal pathogen strain & \\
\hline
\end{tabular}

${ }^{a} \mathrm{Tc}^{r}, \mathrm{Crm}^{r}, \mathrm{Erm}^{r}=$ tetracycline-, chloramphenicol-, and erythromycin-resistance genes.

A
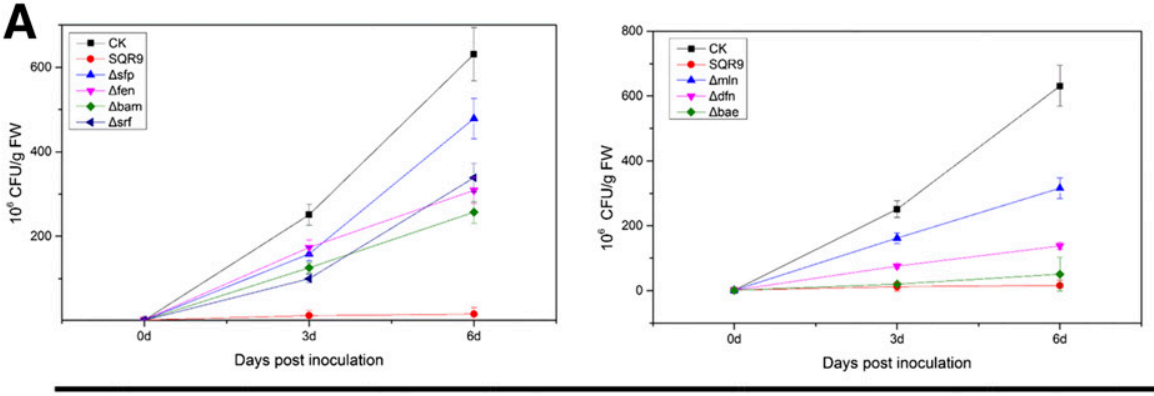

\begin{tabular}{ccccc}
\hline & CK & SQR9 & $\Delta s f p$ & $\Delta f$ \\
\hline 0d & a & a & a & \\
3d & c & a & bc & bc \\
6d & e & a & d & \\
\hline
\end{tabular}

B
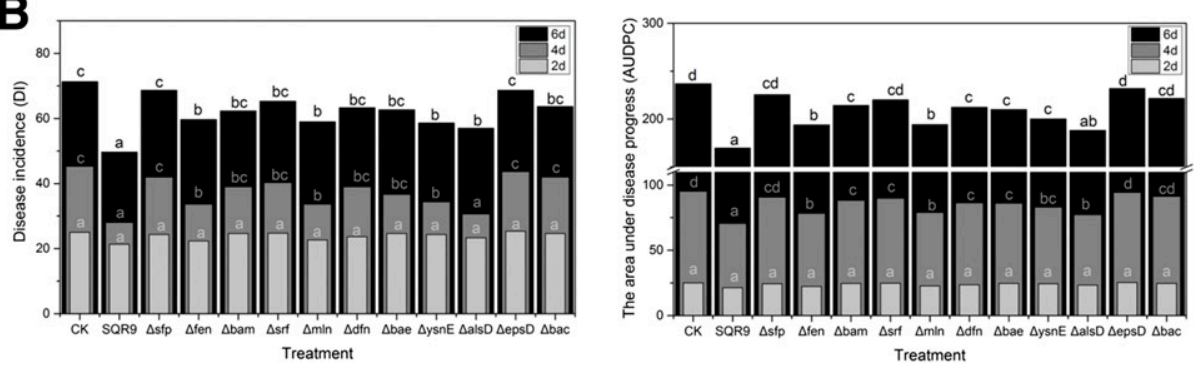

Fig. 4. Disease incidence in Arabidopsis after treatment with SQR9 or its mutants. Five-week-old seedlings were inoculated with SQR9 or its mutants. Four days later, leaves were injected with Pseudomonas syringae pv. tomato DC3000 or Botrytis cinerea. A, Growth curves of DC3000 in the leaves of Arabidopsis inoculated with SQR9 or mutant strains and the statistical analysis. Different letters indicate significant differences between samples $(P \leq 0.05)$. B, Disease severity of Botrytis cinerea in plants inoculated with SQR9 or mutant strains. Symptoms were scored at 2, 4, and 6 days postinoculation. Disease incidence and the area under the disease-progress curve were calculated following the method described previously (Jeger and Viljanen-Rollinson 2001; Madden and Hughes 1999). Different letters indicate significant difference between samples $(P \leq 0.05)$. The results are means of 12 independent experiments. 
signaling genes upon inoculation with SQR9 wild type and elicitor mutants was analyzed using a quantitative reverse transcription-polymerase chain reaction (qRT-PCR) approach at 6 hpi (Supplementary Table S5). The contribution of each elicitor to the transcription of plant defense genes was calculated by dividing the reduced gene transcription of a plant inoculated with the elicitor mutant to that of a plant inoculated with wild-type SQR9 (Fig. 5). The SQR9 mutant $\Delta s f p$, deficient of all antibiotic production, showed a large decrease of 70 to $90 \%$ in the activation of transcription of the measured defense genes (Fig. 5). The lipopeptide antibiotic fengycin was effective in inducing both the SA- and JA-signaling pathways, especially in inducing the transcription of PR2 and COI1. A deficiency of fengycin caused more than a $70 \%$ decrease of upregulation of PR2 and COI1 by SQR9. Bacillomycin D and bacillaene showed a broad range but weak contribution (no more than $50 \%$ ) to all tested defense genes. Surfactin showed a 60 to $70 \%$ contribution to PR5, NPR1, AOS1, MYC2, HEL/PR4, CHIB, and PDF1.2. For polyketide antibiotics, macrolactin showed a 60 to $70 \%$ contribution to PR2, PR5, HEL/PR4, and PDF1.2 and a 50\% contribution to CHIB, ERF1, and AOS1. Difficidin showed an $80 \%$ contribution to ERF1. The dipeptide bacilycin showed a great contribution to the ETsignaling pathway. A deficiency of bacilycin production caused more than an $80 \%$ reduction of the enhancement of HEL/PR4 and ERF1 by SQR9. Moreover, bacilycin showed more than a 50\% contribution to all the tested genes except CHIB and COI1. For the nonantibiotic elicitors, the volatile compound 2,3-butanediol showed an $80 \%$ contribution to PR2 and HEL/PR4 and more than a $60 \%$ contribution to PDF1.2. Reduced phytohormone IAA production $(\triangle y s n E)$ caused an $80 \%$ decrease of the induction of PR5 and $70 \%$ of AOS1. The exopolysaccharide showed more than a $70 \%$ contribution to COI1 and PR5 and an $80 \%$ contribution to PR2.

\section{DISCUSSION}

In the present study, bacterial mutants were used to investigate which bacterial compound serves as the elicitor of plant ISR and which plant signaling pathway is activated by these elicitors. We elaborated the network of these elicitors in B. amyloliquefaciens SQR9 in inducing the systemic resistance of Arabidopsis. A conclusion is that the antibiotics, including lipopeptides (bacillomycin D, fengycin, surfactin), polyketides (bacillaene, macrolactin, difficidin), and the dipeptide bacilysin, play the most important role in triggering plant systemic resistance.

Bacillus spp.-produced surfactin, fengycin, cold shock protein, 2,3-butanediol, acetoin, 2-aminobenzoic acid, and dimethyl disulfide have been identified as elicitors of the plant defense response (Huang et al. 2012; Ongena et al. 2007; Yang et al. 2011; Yi et al. 2016). However, the effect of the lipopeptide bacillomycin $\mathrm{D}$, the polyketide macrolactin $(\mathrm{mln})$, difficidin, bacillaene, and the dipepetide bacilycin on plant systemic resistance has not been reported. We showed that macrolactin is a strong elicitor of plant resistance against DC3000 (Fig. 4A). The dipeptide antibiotic bacilysin, which has shown antibacterial activity against Xanthomonas oryzae and Erwinia amylovora (Wu et al. 2015), showed active participation in regulating Arabidopsis resistance against Botrytis cinerea through the ET and SA signaling pathways.

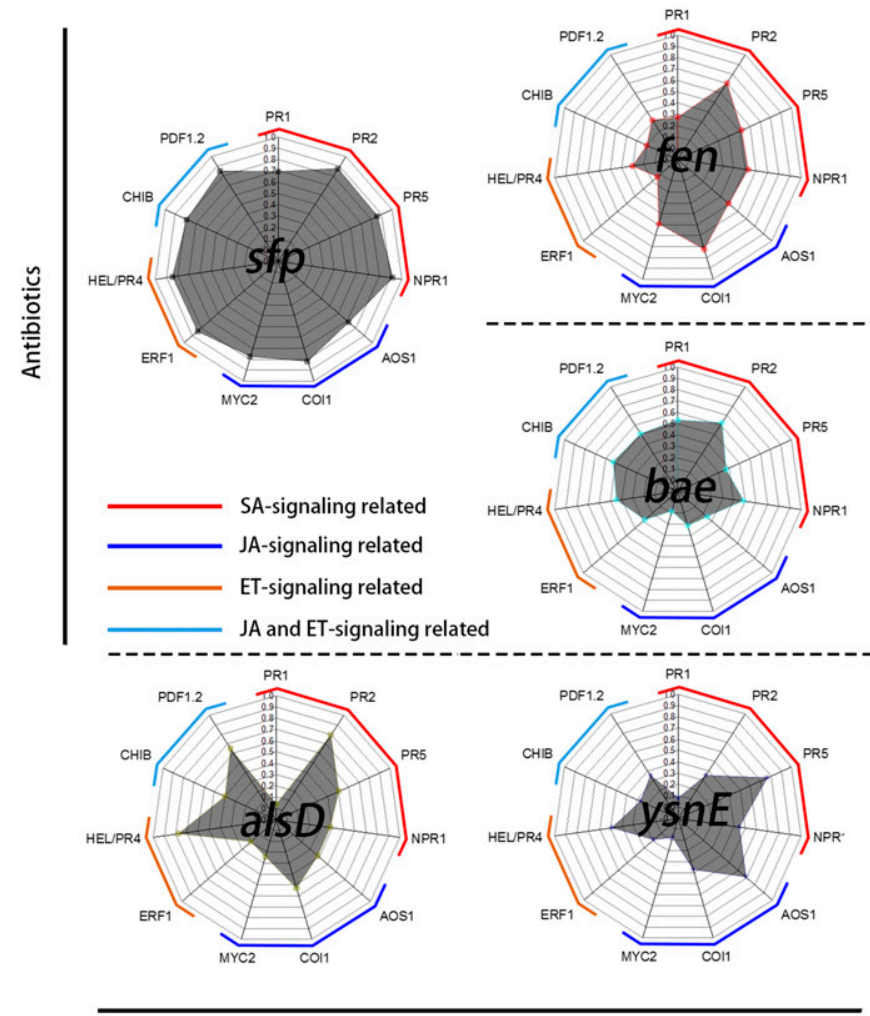

Growth-promoting compounds
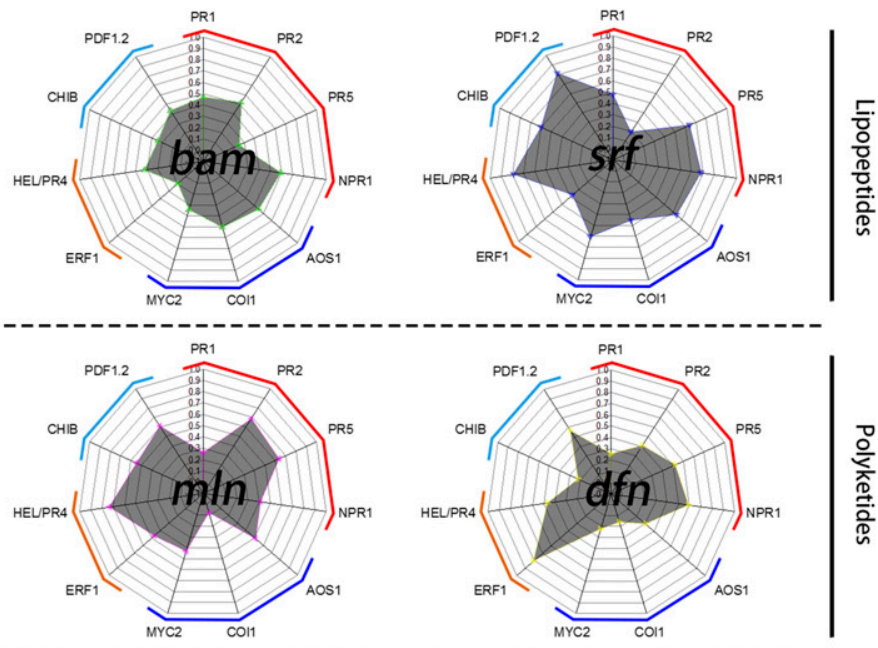

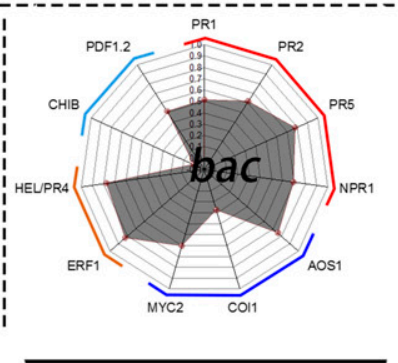

Dipeptide antibiotic

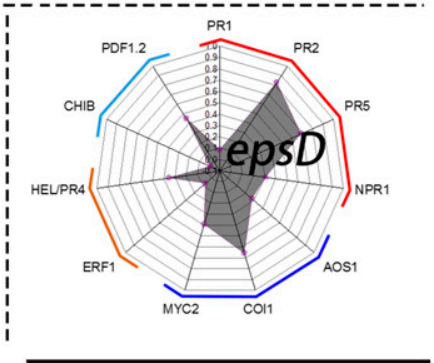

Exopolysaccharide

Fig. 5. Contribution of each elicitor produced by Bacillus amyloliquefaciens SQR9 to the enhanced expression of plant defense genes. The shown values were calculated from the results of real-time quantitative polymerase chain reaction analysis of salicylic acid (SA)-, jasmonic acid (JA)-, and JA/ET-related gene transcript levels in the shoots of Arabidopsis in response to SQR9 and its mutants. Statistical analyses of these transcriptions were performed using analysis of variance. $s f p=\mathrm{SQR} 9$ deficient in producing bacillomycin $\mathrm{D}$, fengycin, surfactin, bacillaene, difficidin, macrolactin, and bacilysin; fen = fengycin; $b a m=$ bacillomycin D; $s r f=$ surfactin; $b a e=$ bacillaene; $m l n=$ macrolactin; $d f n=$ difficidin; $y s n E=$ indole-3-acetic acid; $a l s D=2,3$ - butanediol; $b a c=$ bacilysin; and eps $D=$ exopolysaccharides 
Furthermore, the overall correspondence of these elicitors and the transduction pathways in plants, which has not been studied previously, was comprehensively investigated in this study. Interestingly, we found that induction of the defense genes by each elicitor is relatively specific. For example, bacilysin showed more than an $80 \%$ contribution to the ET signaling pathway but did not participate in the activation of CHIB (Fig. 5). Surfactin, macrolactin, and bacillaene contributed to induction of CHIB. Some of the compounds showed similar effects as previously reported. Surfactin has been reported to be important for bacteria to activate the SA signaling pathway and induce chitinase (CHIB) but showed little effect on 1,3glucanase (PR2) (Farace et al. 2015). Accordingly, we found that deficiency of surfactin production reduced the enhancement of CHIB; however, the transcription enhancement by SQR9 was not completely blocked. One reason is there are two other elicitors (macrolactin and bacillaene) produced by this strain that are involved in the activation of the transcription of CHIB (Fig. 5). A SQR9 mutant deficient in 2,3-butanediol, a kind of volatile organic compound, activated the transcription of PR2 at much lower levels than the wild-type strain did, which is consistent with previous reports (Yi et al. 2016). Exopolysaccharides produced by Burkholderia gladioli IN26 enhanced the expression of PR1a in cucumber (Park et al. 2008); however, in this study, the exopolysaccharides contributed greatly to the enhanced expression of PR2 and PR5 but not that of PR1. Exopolysaccharides synthesized by different bacteria vary greatly in their composition and, hence, in their chemical and physical properties (Flemming and Wingender 2010), which may affect their abilities to induce defense genes.

SQR9-produced macrolactin and fengycin induced stronger plant resistance against $P$. syringae pv. tomato DC3000 but not against Botrytis cinerea, whereas bacilycin induced a stronger resistance against Botrytis cinerea but not against DC3000 (Fig. 4). It is known that Botrytis cinerea is a kind of necrotrophic pathogen, and plant resistance against these pathogens generally depends on the JA/ET signaling pathways (Pieterse et al. 2009); while DC3000 is a hemibiotrophic pathogen, the plant resistance against it generally depends on the SA signaling pathway, although with exceptions (Pieterse et al. 2009). When comparing the effect of macrolactin, fengycin, and bacilycin on plant resistance-related genes, bacilycin showed the strongest effect on the JA/ET signaling pathway genes compared with any other elicitors (Fig. 5), especially for the transcription factor ERF1 and HEL/PR4 (Fig. 5) (Fernández-Calvo et al. 2011; Mao et al. 2016). Macrolactin showed a stronger effect than bacilycin on the induction of CHIB, which is generally recognized to contribute to plant defense through pathogen cell-wall degradation (Pieterse et al. 2009). However, macrolactin was less effective than bacilycin in inducing plant resistance against Botrytis cinerea (Fig. 4B). It indicated that upregulation of CHIB is not necessary for ISR against Botrytis cinerea. However, it is still not clear whether CHIB is effective against different phytopathogens.

Interestingly, it was observed that phytohormone accumulation in distal plant tissue was slower than in local tissue after inoculation with SQR9 (Fig. 2). The transition of signal from local to distal tissue is achieved by a range of mobile chemicals. Methyl salicylate (MeSA) and ET could serve as media in the long distance signaling-transduction in plant (Dempsey and Klessig 2012; Shah and Zeier 2013). This indicated that the time delay of phytohormone enhancement in distal tissue (shoot) could be caused by the signal translocation from root to shoot.

It is known that some of the secondary metabolites exert multiple functions in bacteria besides antagonistic activity and inducing plant resistance. For instance, surfactin has been reported to enhance the biofilm formation of Bacillus subtilis (Aleti et al. 2016). Experiments with pure surfactin showed consistent results with an experiment using a srf mutant (Supplementary Fig. S1), which indicated that the strategy of this study to use a potential elicitor mutant to evaluate their contribution to plant ISR is reliable but these results cannot be turned into the true effect of the chemically purified elicitor compound. Moreover, the correlation analysis between hormone accumulation and gene expression after inoculation with SQR9 and mutants confirmed the correspondence of bacterial genes and plant defense genes and the cross-talk between hormones and signaling pathways (Supplementary Fig. S2).

In conclusion, plant-beneficial rhizobacterium SQR9 produced multiple elicitors to induce systemic resistance in Arabidopsis against DC3000 and Botrytis cinerea, and these lipopeptides, polyketides, dipepetide antibiotics, 2,3-butandiol, and exopolysaccharides played a major role to the ISR. Elicitors have specific effects on the induction of plant defense pathways and against different phytopathogens. Further investigation of the complex crosstalk between the multiple elicitors and the signaling pathways are needed to provide further insights into the interactions between beneficial rhizobacteria and plants.

\section{MATERIALS AND METHODS}

\section{Growth conditions of plants and microbes.}

The Col-0 seeds were surface-sterilized with $75 \%$ (vol/vol) ethanol and, then, with $2 \%$ ( $\mathrm{vol} / \mathrm{vol}$ ) $\mathrm{NaClO}$, after which they were placed in petri dishes containing $1 / 2$ Murashige and Skoog (MS) medium with $2 \%$ (wt/vol) sucrose and $0.8 \%$ (wt/vol) agar. After vernalizing for 2 days at $4^{\circ} \mathrm{C}$ in darkness, plants were grown under $16-\mathrm{h}$ light and 8 -h dark cycles at $22^{\circ} \mathrm{C}$. Ten days later, seedlings were transferred to new petri dishes containing $1 / 2$ MS medium with $2 \%(\mathrm{wt} / \mathrm{vol})$ sucrose and $1.5 \%(\mathrm{wt} / \mathrm{vol})$ agar for inoculation with SQR9 or its mutants. For assessing the resistance of Arabidopsis to DC3000 and Botrytis cinerea, 10day-old seedlings were transferred to a growth chamber with a vermiculite-peat soil mixture and were allowed to grow for 5 weeks.

Bacillus amyloliquefaciens SQR9 (China General Microbiology Culture Collection Center accession number 5808), including wild type and mutants (Table 1), was grown in LuriaBertani (LB) liquid medium at $37^{\circ} \mathrm{C}$ and $170 \mathrm{rpm}$ to an optical density at $600 \mathrm{~nm}\left(\mathrm{OD}_{600}\right)$ of 1.0. Subsequently, bacterial cells were pelleted by centrifugation and were suspended to $5 \times 10^{8}$ $\mathrm{CFU} / \mathrm{ml}$ for use. DC3000 was grown in King's B (KB) liquid medium, containing $50 \mathrm{mg}$ of rifampicin per liter, at $28^{\circ} \mathrm{C}$ and $170 \mathrm{rpm}$ for $18 \mathrm{~h}$. Subsequently, bacterial cells were pelleted by centrifugation and were suspended in $10 \mathrm{mM} \mathrm{MgCl}_{2}$ to $10^{6} \mathrm{CFU} / \mathrm{ml}$. Botrytis cinerea was grown on petri dishes filled with potato dextrose agar medium at $28^{\circ} \mathrm{C}$ for 10 days. Spores were collected by washing the colony with sterile water. The concentration was evaluated under a microscope using a counter plate.

\section{Construction of SQR9 mutants.}

To disrupt 2,3-butanediol synthesis in SQR9, the alsD gene was completely deleted by double crossover (Yan et al. 2008). The erythromycin-resistant cassette was obtained from the plasmid pAX01. Two partial sequence fragments of the alsD gene were amplified from SQR9 DNA. Then, the recombinant fragments were fused and transformed into the SQR9 strain to generate the $\triangle a l s D$ mutation. The transformants were selected on LB agar plates containing erythromycin. After that, sequencing of the transformants was performed to confirm that the gene was completely knocked out. Mutant strains $\Delta b a e, \Delta m \ln , \Delta d f n 2$, 
$\Delta b a c 3, \Delta e p s D$, and $\Delta a l s D$ were constructed in the same manner, using chloramphenicol resistance as the screening marker.

For all these mutants of polypeptides and polyketides, high performance liquid chromatography detection was performed to confirm that the syntheses of the antibiotics were completely blocked (Li et al. 2014; Xu et al. 2013).

\section{Measurement of SA, JA, and ET contents in plant.}

Ten-day-old seedlings of wild-type Arabidopsis were planted on new petri dishes containing $1 / 2 \mathrm{MS}$ medium with $2 \%$ (wt/vol) sucrose and $1.5 \%(\mathrm{wt} / \mathrm{vol})$ agar. A 5- $\mu \mathrm{l} \mathrm{SQR} 9$ suspension $\left(\mathrm{OD}_{600}=\right.$ 1.0) was separately inoculated onto the petri dishes. At 1,2 , 4, and 6 dpi, plant tissues (shoots and roots) were collected and were ground in $1.5 \mathrm{ml}$ of sodium phosphate buffer $(\mathrm{pH} \mathrm{7.0)}$ and were then centrifuged at $13,500 \times g$ for $10 \mathrm{~min}$, after which the supernatants were collected for the detection of SA, JA, and ET. Twelve biological replicates were included per treatment.

Measurements were performed using an enzyme linked immunosorbent assay (ELISA) (Lengton Bioscience Co., Ltd, Shanghai, China). Supernatant and horseradish peroxidase conjugate reagent $(50 \mu \mathrm{l}$, each) were added to each well of the ELISA kit plate. The wells were incubated at $37^{\circ} \mathrm{C}$ for $60 \mathrm{~min}$ and were then washed five times. Afterward, color reactions were performed for $15 \mathrm{~min}$ at $37^{\circ} \mathrm{C}$ in darkness. Absorbance at $450 \mathrm{~nm}$ was then measured, and the concentration was calculated based on the standard curve.

\section{Extraction of RNA from plant tissue.}

Ten-day-old seedlings were transferred to new petri dishes containing $1 / 2$ MS medium for 2 days. Afterward, $5 \mu$ of suspension of SQR9 or its mutants was inoculated onto the $1 / 2 \mathrm{MS}$ medium. After $6 \mathrm{~h}$, RNA was extracted from the shoots of Arabidopsis in each treatment. The plant tissue was flashfrozen in liquid nitrogen and was then ground. The extraction of RNA was performed using the Qiagen RNeasy plant mini kit (Qiagen, Valencia, CA, U.S.A.). The extracted RNA was evaluated on a $1 \%$ agarose gel, and the concentration and quality $\left(\mathrm{A}_{260} / \mathrm{A}_{280}\right)$ were determined by a NanoDrop ND-2000 spectrophotometer (NanoDrop, Wilmington, DE, U.S.A.).

\section{Quantification of the transcription of defense-related genes.}

qRT-PCR was performed using a Prime Script RT reagent kit (Takara Biotechnology Co., Ltd., Dalian, China) with an ABI7500 Cycler (Applied Biosystems, Foster City, CA, U.S.A.). Their action solution was prepared with SYBR Premix EXTaq (Takara Biotechnology Co., Ltd.). The reaction system $(20 \mu \mathrm{l})$ included $10 \mu \mathrm{l}$ of SYBR Premix Ex Taq (2x), $0.4 \mu \mathrm{l}$ of PCR forward primer $(10 \mu \mathrm{M}), 0.4 \mu \mathrm{l}$ of PCR reverse primer $(10 \mu \mathrm{M})$, $0.4 \mu \mathrm{l}$ of rhodamine $\mathrm{X}$ reference dye $(50 \times), 2 \mu \mathrm{l}$ of DNA sample, and $6.8 \mu \mathrm{l}$ of double distilled $\mathrm{H}_{2} \mathrm{O}$. Thermal conditions were as follows: $30 \mathrm{~s}$ at $95^{\circ} \mathrm{C}$ for initial denaturation and 40 cycles of $5 \mathrm{~s}$ at $95^{\circ} \mathrm{C}$, followed by $34 \mathrm{~s}$ at $60^{\circ} \mathrm{C}$. The transcription levels of $P R 1$ (encoding pathogenesis-related protein 1), $P R 2$ (encoding $\beta$-1,3-glucanase), PR5 (encoding thaumatin-like proteins), $n p r 1$ (encoding regulatory protein NPR1), myc2 (encoding transcription factor MYC2), coil (encoding coronatine-insensitive protein 1), aos (encoding allene oxide synthase), hel (encoding hevein-like protein), erfl (encoding ET-responsive transcription factor 1B), etrl (encoding ET receptor 1), chiB (encoding basic chitinase), and pdf1.2 (encoding plant defensin) were measured. The Arabidopsis actin gene was used as an internal reference. For these genes, primers were listed in Supplementary Table S6. Cycling threshold $(\mathrm{Ct})$ values, which represent relative expression, were used for further analysis.

To correlate the SQR9 metabolites with each plant defense gene, qRT-PCR was performed to determine how these mutant strains affect the transcription of defense genes. The RNA of the shoot tissue of plants inoculated with SQR9 or mutant strains was extracted. Using the effect of wild-type SQR9 on the gene transcription in Arabidopsis as $100 \%$ efficiency, the lost activity of each mutant strain (contribution of the elicitor for enhancing the transcription of the defense gene against SQR9) was calculated for each plant defense gene, using the following formula to show the contribution of each compound. Statistical analyses of these transcriptions were performed using analysis of variance. The transcription levels of the defense gene in Arabidopsis inoculated with wild-type SQR9, Arabidopsis inoculated with an elicitor-deficient mutant of SQR9, and uninoculated Arabidopsis at $6 \mathrm{hpi}$ were denoted with Q(SQR9WT), Q(SQR9mutant), and Q(CK), respectively.

Contribution $=$

$$
\frac{(Q(S Q R 9 W T)-Q(C K))-(Q(S Q R 9 \text { mutant }))-(Q(C K))}{Q(S Q R 9 W T)-Q(C K)}
$$

\section{Disease assays.}

Ten-day-old seedlings of wild-type Arabidopsis were transplanted into $200-\mathrm{ml}$ pots filled with vermiculite-peat soil mixture and were allowed to grow for 5 weeks. Seedlings were inoculated with $4 \mathrm{ml}$ of SQR9 $\left(\mathrm{OD}_{600}=1.0\right)$ or its mutants.

Leaf injection of $P$. syringae pv. tomato DC3000 at $10^{6}$ $\mathrm{CFU} / \mathrm{ml}$ was performed 4 days after inoculation with SQR9 or its mutants. Ten millimolar $\mathrm{MgCl}_{2}$ was injected as a mock treatment. The population of DC3000 was measured after inoculation for 3 and 6 days. Each leaf sample was washed with sterile water, was soaked in $75 \%$ (vol/vol) ethanol for $30 \mathrm{~s}$ for surface sterilization, was washed in sterile distilled water three times, and was then extracted, using grinding beads and $1 \mathrm{ml}$ of $\mathrm{MgCl}_{2}(10 \mathrm{mM})$ in a tissue grinder. Subsequently, appropriate dilutions were plated onto $\mathrm{KB}$ agar supplemented with $50 \mathrm{mg}$ of rifampicin per liter and were incubated at $28^{\circ} \mathrm{C}$ for $24 \mathrm{~h}$. Afterward, rifampicin-resistant DC3000 colonies on plates were counted, and the DC3000 density in the leaves was thus determined and expressed as CFU per gram of leaf fresh weight. This experiment was repeated 12 times.

At 4 dpi with SQR9 or the mutants, 5-week-old seedlings of Arabidopsis were sprayed with $5 \times 10^{5}$ spores of Botrytis cinerea per milliter. Water was included as a mock treatment. Symptoms were scored at 2, 4, and 6 dpi with Botrytis cinerea. The AUDPC of each leaf was measured, the disease incidence was calculated according to the incidence area based on previously described methods (Jeger and Viljanen-Rollinson 2001; Madden and Hughes 1999).

\section{LITERATURE CITED}

Aleti, G., Lehner, S., Bacher, M., Compant, S., Nikolic, B., Plesko, M., Schuhmacher, R., Sessitsch, A., and Brader, G. 2016. Surfactin variants mediate species-specific biofilm formation and root colonization in Bacillus. Environ. Microbiol. 18:2634-2645.

Cao, Y., Zhang, Z., Ling, N., Yuan, Y., Zheng, X., Shen, B., and Shen, Q. 2011. Bacillus subtilis SQR9 can control Fusarium wilt in cucumber by colonizing plant roots. Biol. Fert. Soils 47:495-506.

Cawoy, H., Debois, D., Franzil, L., De Pauw, E., Thonart, P., and Ongena, M. 2015. Lipopeptides as main ingredients for inhibition of fungal phytopathogens by Bacillus subtilis/amyloliquefaciens. Microb. Biotechnol. 8:281-295.

Chen, L., Liu, Y., Wu, G., Veronican Njeri, K., Shen, Q., Zhang, N., and Zhang, R. 2016. Induced maize salt tolerance by rhizosphere inoculation of Bacillus amyloliquefaciens SQR9. Physiol. Plant. 158:34-44.

Chen, L., Liu, Y., Wu, G., Zhang, N., Shen, Q., and Zhang, R. 2017. Beneficial rhizobacterium Bacillus amyloliquefaciens SQR9 induces plant salt tolerance through spermidine production. Mol. Plant-Microbe Interact. 30:423-432.

Dempsey, D. A., and Klessig, D. F. 2012. SOS-Too many signals for systemic acquired resistance? Trends Plant Sci. 17:538-545.

Farace, G., Fernandez, O., Jacquens, L., Coutte, F., Krier, F., Jacques, P., Clément, C., Barka, E. A. I. T., Jacquard, C., and Dorey, S. 2015. Cyclic 
lipopeptides from Bacillus subtilis activate distinct patterns of defence responses in grapevine. Mol. Plant Pathol. 16:177-187.

Fernández-Calvo, P., Chini, A., Fernández-Barbero, G., Chico, J.-M., Gimenez-Ibanez, S., Geerinck, J., Eeckhout, D., Schweizer, F., Godoy, M., Franco-Zorrilla, J. M., Pauwels, L., Witters, E., Puga, M. I., PazAres, J., Goossens, A., Reymond, P., De Jaeger, G., and Solano, R. 2011. The Arabidopsis bHLH transcription factors MYC3 and MYC4 are targets of JAZ repressors and act additively with MYC2 in the activation of jasmonate responses. Plant Cell 23:701-715.

Flemming, H.-C., and Wingender, J. 2010. The biofilm matrix. Nat. Rev. Microbiol. 8:623-633.

Glazebrook, J. 2005. Contrasting mechanisms of defense against biotrophic and necrotrophic pathogens. Annu. Rev. Phytopathol. 43:205-227.

Huang, C. J., Tsay, J. F., Chang, S. Y., Yang, H. P., Wu, W. S., and Chen, C. Y. 2012. Dimethyl disulfide is an induced systemic resistance elicitor produced by Bacillus cereus C1L. Pest Manag. Sci. 68:1306-1310.

Iavicoli, A., Boutet, E., Buchala, A., and Métraux, J. P. 2003. Induced systemic resistance in Arabidopsis thaliana in response to root inoculation with Pseudomonas fluorescens CHA0. Mol. Plant-Microbe Interact. 16:851-858.

Jeger, M. J., and Viljanen-Rollinson, S. L. H. 2001. The use of the area under the disease-progress curve (AUDPC) to assess quantitative disease resistance in crop cultivars. Theor. Appl. Genet. 102:32-40.

Kloepper, J. W. 1993. Plant growth promoting rhizobacteria as biological control agents. Pages 255-274 in: Soil Microbial Ecology. B. Metting, ed. Marcel Dekker, Inc., New York.

Li, B., Li, Q., Xu, Z., Zhang, N., Shen, Q., and Zhang, R. 2014. Responses of beneficial Bacillus amyloliquefaciens SQR9 to different soilborne fungal pathogens through the alteration of antifungal compounds production. Front. Microbiol. 5:636.

Liu, Y., Chen, L., Wu, G., Feng, H., Zhang, G., Shen, Q., and Zhang, R. 2017. Identification of root-secreted compounds involved in the communication between cucumber, the beneficial Bacillus amyloliquefaciens, and the soilborne pathogen Fusarium oxysporum. Mol. Plant-Microbe Interact. 30:53-62.

Liu, Y., Chen, L., Zhang, N., Li, Z., Zhang, G., Xu, Y., Shen, Q., and Zhang, R. 2016. Plant-microbe communication enhances auxin biosynthesis by a root-associated bacterium, Bacillus amyloliquefaciens SQR9. Mol. Plant-Microbe Interact. 29:324-330.

Liu, Y., Zhang, N., Qiu, M., Feng, H., Vivanco, J. M., Shen, Q., and Zhang, R. 2014. Enhanced rhizosphere colonization of beneficial Bacillus amyloliquefaciens SQR9 by pathogen infection. FEMS Microbiol. Lett. 353:49-56.

Madden, L. V., and Hughes, G. 1999. Sampling for plant disease incidence. Phytopathology 89:1088-1103.

Mao, J. L., Miao, Z. Q., Wang, Z., Yu, L. H., Cai, X. T., and Xiang, C. B. 2016. Arabidopsis ERF1 mediates cross-talk between ethylene and auxin biosynthesis during primary root elongation by regulating ASA1 expression. PLoS Genet. 12:1-20.

Meziane, H., Van Der Sluis, I., van Loon, L. C., Höfte, M., and Bakker, P. A. 2005. Determinants of Pseudomonas putida WCS358 involved in inducing systemic resistance in plants. Mol. Plant Pathol. 6:177-185.

Ongena, M., Jourdan, E., Adam, A., Paquot, M., Brans, A., Joris, B., Arpigny, J. L., and Thonart, P. 2007. Surfactin and fengycin lipopeptides of Bacillus subtilis as elicitors of induced systemic resistance in plants. Environ. Microbiol. 9:1084-1090.
Park, K., Kloepper, J. W., and Ryu, C.-M. 2008. Rhizobacterial exopolysaccharides elicit induced resistance on cucumber. J. Microbiol. Biotechnol. 18:1095-1100.

Pieterse, C. M. J., Leon-Reyes, A., Van der Ent, S., and Van Wees, S. C. M. 2009. Networking by small-molecule hormones in plant immunity. Nat. Chem. Biol. 5:308-316.

Pieterse, C. M. J., Zamioudis, C., Berendsen, R. L., Weller, D. M., Van Wees, S. C. M., and Bakker, P. A. H. M. 2014. Induced systemic resistance by beneficial microbes. Annu. Rev. Phytopathol. 52:347-375.

Ryu, C.-M., Farag, M. A., Hu, C.-H., Reddy, M. S., Kloepper, J. W., and Paré, P. W. 2004. Bacterial volatiles induce systemic resistance in Arabidopsis. Plant Physiol. 134:1017-1026.

Ryu, C.-M., Hu, C., Reddy, M. S., and Kloepper, J. W. 2003. Different signaling pathways of induced resistance by rhizobacteria in Arabidopsis thaliana against two pathovars of Pseudomonas syringae. New Phytol. 160:413-420

Schuhegger, R., Ihring, A., Gantner, S., Bahnweg, G., Knappe, C., Vogg, G., Hutzler, P., Schmid, M., Van Breusegem, F., Eberl, L., Hartmann, A., and Langebartels, C. 2006. Induction of systemic resistance in tomato by $\mathrm{N}$-acyl-L-homoserine lactone-producing rhizosphere bacteria. Plant Cell Environ. 29:909-918.

Shah, J., and Zeier, J. 2013. Long-distance communication and signal amplification in systemic acquired resistance. Front. Plant Sci. 4:30.

Shao, J., Xu, Z., Zhang, N., Shen, Q., and Zhang, R. 2015. Contribution of indole-3-acetic acid in the plant growth promotion by the rhizospheric strain Bacillus amyloliquefaciens SQR9. Biol. Fertil. Soils 51:321-330.

Sharifi, R., and Ryu, C.-M. 2016. Are bacterial volatile compounds poisonous odors to a fungal pathogen Botrytis cinerea, alarm signals to Arabidopsis seedlings for eliciting induced resistance, or both? Front. Microbiol. 7:196.

Wang, N., Liu, M., Guo, L., Yang, X., and Qiu, D. 2016. A novel protein elicitor (PeBA1) from Bacillus amyloliquefaciens NC6 induces systemic resistance in tobacco. Int. J. Biol. Sci. 12:757-767.

Wu, L., Wu, H., Chen, L., Yu, X., Borriss, R., and Gao, X. 2015. Difficidin and bacilysin from Bacillus amyloliquefaciens FZB42 have antibacterial activity against Xanthomonas oryzae rice pathogens. Sci. Rep. 5:12975.

Xu, Z., Shao, J., Li, B., Yan, X., Shen, Q., and Zhang, R. 2013. Contribution of bacillomycin D in Bacillus amyloliquefaciens SQR9 to antifungal activity and biofilm formation. Appl. Environ. Microbiol. 79:808-815.

Yan, X., Yu, H. J., Hong, Q., and Li, S. P. 2008. Cre/lox system and PCRbased genome engineering in Bacillus subtilis. Appl. Environ. Microbiol. 74:5556-5562.

Yang, S. Y., Park, M. R., Kim, I. S., and Kim, Y. C. 2011. 2-Aminobenzoic acid of Bacillus sp. BS107 as an ISR determinant against Pectobacterium carotovorum subsp. carotovotrum SCC1 in tobacco. Eur. J. Plant Pathol. 129:371-378

Yi, H.-S., Ahn, Y. R., Song, G. C., Ghim, S.-Y., Lee, S., Lee, G., and Ryu, C.-M. 2016. Impact of a bacterial volatile 2,3-butanediol on Bacillus subtilis rhizosphere robustness. Front. Microbiol. 7:993.

Yi, H.-S., Yang, J. W., and Ryu, C.-M. 2013. ISR meets SAR outside: Additive action of the endophyte Bacillus pumilus INR7 and the chemical inducer, benzothiadiazole, on induced resistance against bacterial spot in field-grown pepper. Front. Plant Sci. 4:122. 